\title{
Desarrollo de la teoria de sistemas en las Ciencias Sociales
}

\author{
Marcelo Arnold
}

A partir de la segunda década de este siglo empiezan a proliferar y difundirse paulatinamente, en nuestro lenguaje científico, conceptos y relaciones provenientes de la denominada teoria o perspectiva de sistemas. Esta nueva orientación ancla sus orígenes en disciplinas tradicionales tales como la biología y la etnología. De ellas emerge a partir de la observación de los problemas no resueltos en sus campos fenoménicos -el problema de la vida y el de la dinámica sociocultural, respectivamente - una noción de totalidad, la cual prontamente se aplica al análisis de organismos, sociedades y culturas, en términos de evaluarlas en cuanto entidades cualitativamente distintas a las partes o elementos que las componen y por ende propietarias de ciertas condiciones y potencialidades, altamente improbables a la luz de los recursos explicativos tradicionales. En particular, importante es la conciencia de la inaplicabilidad de las leyes fisicoquímicas y de las perspectivas atomistas en las ciencias sociales. Todos estos problemas se han encarado u tilizando el concep to de sistema ${ }^{1}$.

En la Etnología y Antropología Social los estudios de las instituciones socioculturales ganan su coherencia cuando éstas se estudian en relación al todo del cual formarían parte. Esta aproximación es una consecuencia de la optimización de los recursos explicativos y predictivos disponibles por investigadores tales como: Malinowski (1884-1942) y Radcliffe-Brown (1881-1955) y, en general, de toda la matriz de pensamiento funcionalista clásico. En la práctica, los funcionalistas hicieron una apropiación y uso implícito de nociones propiamente sistémicas. Para esta línea de pensamiento los sistemas socioculturales son concebidos en cuanto unidades compuestas por un conjunto de

\footnotetext{
${ }^{1}$ La palabra sistema proviene del griego y significa algo así como combinación, composi ción, poner junto. Esa etimología ha jugado un importante rol en las definiciones posteriores.
} 
elementos y sus atributos, los cuales están unidos mediante relaciones interactivas sujetas a algún nivel de regularidad y de estabilidad. Bajo estos puntos de vista pusieron, en sus estudios, especial énfasis en las nociones y referentes empíricos que destacaban la interrelación e interdependencia de las instituciones culturales, descuidando, en compensación, los estudios acerca del cambio y del conflicto.

Por otro lado, los avances en la biología y sus métodos de estudio fueron retomados por Comte (1798-1857) y Spencer (1820-1903) aplicándose al estudio de la sociedad, iniciando con ello una profunda resonancia entre el pensamiento social y las concepciones biológicas. No se puede dejar de mencionar en este plano al mismo Durkheim, quien, adelantándose con mucho a los conocimientos de su época, le atribuye a la sociedad -y a la cultura - el carácter de realidad comprensible en sí misma -sui generis-, idea que está fuertemente emparentada con los más recientes avances en la teoría que se aplica a los sistemas socioculturales.

Por cierto, la búsqueda de predecesores en el análisis de sistemas es tarea inacabable. El mismo Durkheim (1858-1917) le atribuye a Montesquieu (1689-1755) la primera aplicación coherente de las nociones de interdependencia e interrelación al interior de las sociedades. Pero sin ir más lejos Aristóteles no sólo había intuido el problema, sino que ya había trabajado con estas ideas en el campo de lo social. Así también encontramos esbozos de perspectivas sistémicas en el pensamiento filosófico escolástico, que fue a su vez retomado del pensamiento griego.

Pero es en estas últimas décadas cuando se expande e intensifica fuertemente en las ciencias humanas y sociales el uso - y abuso- de una terminología que proviene de la teoría general de sistemas (TGS), la cual ha pasado de ser una moda intelectual a constituirse en un expansivo paradigma que se extiende por todas las áreas del quehacer de nuestras disciplinas. La causas de este acelerado desarrollo pueden ser interpretadas como una reacción ante dos grandes y complejas dificultades, las cuales no fueron convenientemente resueltas por otras teorías concurrentes, a saber:

a) una creciente dificultad para la comunicación científica, consecuencia de la progresiva especialización temática y conceptual de las diferentes disciplinas, ante lo cual una teoria general, como la de sistemas, ofrece un lenguaje común, sentando con ello las bases para una integración cientifica, o al menos para una potencialmente fecunda interd isciplinariedad. En este sentido el aporte de la teoría de sistemas es visto en términos de una especie de metalenguaje que facilita la comunicación intra e interdisciplinaria $y$,

b) la capacidad que entregaria la teoria de sistemas, para el caso específico de las ciencias humanas y sociales, de estudiar los diversos ámbitos de la realidad sociocultural sin perder la riqueza de la visión de conjunto. Con ella pueden ser contrarrestadas las tendencias que inciden en la proliferación de teorías parciales de alcance medio 
(Merton), en desmedro de una teoría general que las haga complementarias y sus hallazgos acoplables. En este sentido la teoría de sistemas es concebida en términos de un horizonte definido que genera y sistematiza información dando cuenta de totalidades y no de fragmentos.

Visto de esta manera, el auge de la teoría de sistemas respondería al agotamiento de las perspectivas parciales y de la especialización científica frente a las nuevas complejidades que el mismo desarrollo científico ha ido originando. Sin embargo, el problema a resolver acompaña a la misma teoría de sistemas. Esta se ha compartimentalizado demasiado y con ello se han multiplicado las introducciones a tan compleja perspectiva. Todo ello nos invita a exponer algunas ideas en torno al desarrollo general que ha seguido la noción de sistema y los enfoques sociológicos y antropológicos que a partir de ella se han ido derivando ${ }^{2}$.

Los cambios en la teoría de sistemas, al ser reconstituidos en etapas, se expresan como un acrecentamiento continuo de sus niveles de complejidad, lo cual se proyecta en un constante incremento en su cobertura y capacidad de análisis. En la actualidad se está en condiciones de intentar la aplicación de esta teoría a sí misma, en este caso a su propio desarrollo - el cual está ligado a los procesos mismos de construcción de sistemas-, cuyo punto de referencia es un proceso de diferenciación, en términos de la relación dinámica que se establece a partir de nuevas distinciones entre sistema y entorno, ahora al interior del propio sistema científico. Desde este punto de vista pueden ser claramente perfiladas las diferentes concepciones que han acompañado al desarrollo de esta teoría.

En una estrecha síntesis se puede afirmar que las primeras nociones de sistema se aplicaron tomando como base la idea de totalidad, cuyo principio metodológico implicaba que determinados fenómenos sólo podrían ser estudiados desde una perspectiva holística. En la práctica, ello se proyectó en concepciones de sistemas en las que estaba ausente una consideración substantiva del entorno y su importancia para la constitución de éstos. Problema central en esta etapa es la fijación de los límites de un sistema. Posteriormente nuevos avances son alcanzados cuando se empieza a destacar la diferencia de complejidad que permite delimitar un sistema de su entorno. El entorno es observado de diversas maneras, ya sea como fuente de perturbaciones y de desequilibrios o como una fuente inagotable de los recursos que posibilitan la sobrevivencia del sistema. En ambos casos se maneja la idea de dependencia del sistema con respecto a su entorno. En un siguiente paso los estudiosos, en este campo, empiezan a percibir y concebir todo sistema en su condición de propietario de algunos mecanismos selectivos, a través de los cuales se desarrollaría una activa

\footnotetext{
${ }^{2}$ Un excelente análisis del desarrollo de la moderna teoría de sistemas en las ciencias sociales se encuentra en el artículo de Darío Rodríguez Teoría de sistemas. situación actual (1985 op. cit.).
} 
capacidad de respuesta frente a su ambiente. Esta última idea es recogida, en lo sucesivo, por nuevos avances en la teoría, en especial cuando se empiezan a destacar los márgenes de autonomía y la capacidad de autoorganización con que cuentan los sistemas. En definitiva, en estos últimos años son incorporadas a la teoría de sistemas las nociones de autorreferencialidad, autoobservación, reflexividad y, finalmente, la de autopoiesis.

Desde el punto de vista de un observador interesado en las reconstrucciones evolutivas, el paso de una concepción sistémica a otra puede ser visto como un proceso dialéctico -una Aufhebung en el sentido hegeliano--, en el cual un nuevo nivel de desarrollo asume y a la vez sobrepasa a los que lo preceden, los cuales terminan por evaluarse como insuficientes para abordar las mismas realidades que contribuyeron a iluminar. En otras palabras, se va reflejando en un aumento de la complejidad interna de la teoría $y$, por lo tanto, en un incremento significativo de su capacidad de análisis y de su aplicación a una cada vez mayor diversidad de fenómenos. Algunas de estas etapas, realmente, pueden ser percibidas como verdaderos cambios paradigmá ticos o revoluciones científicas, en el sentido establecido por Kuhn (1973). Las teorias parciales y los métodos son fuertemente removidos, los fenómenos e incluso la ciencia misma pasan a ser vistos desde otra perspectiva.

Para las ciencias sociales los cambios a que antes aludíamos pueden ser reconstruidos en su carácter de enfoques, los que no se superan unos a otros, en el sentido de eliminarse, sino más bien tienden a coexistir y a competir entre ellos. Estos van desde una concepción de sistema probablemente ontológica que recurre con frecuencia a esquemas teleológicos y que presupone, de una manera explícita o implícita, algún tipo de estructura a priori o que se representan a sí mismas como enfoques puramente analíticos, hasta nociones avanzadas de concepciones autopoiéticas aplicadas a los sistemas socioculturales.

En este transcurrir puede ser claramente establecido, como constante, que los avances y cambios más significativos, para las ciencias sociales, en la teoría de sistemas han sido originados en investigaciones en el campo de la biología. Primero Ludwig von Bertalanffy, que por la década de los veinte introduce la noción de sistemas abiertos (1950), abriendo paso con ello a la comprensión de la compleja red de relaciones que unen a los sistemas con sus ambientes, y más recientemente Humberto Maturana, que inaugura una concepción de los sistemas en términos de la autopoiesis (1973). De una manera más rezagada, estos aportes han sido acompañados por modificaciones en las teorías sociológicas y son aún poco perceptibles en la teoría antropológica, salvo en lo que se ha denominado Ecología Cultural (Qu1roz), aunque algunos avances pueden ser apreciados. En la Conferencia Mundial de Antropología, realizada en Houston, Texas, por 1977, uno de los seis paneles organizados para la discusión de temas de teoría se focalizó en la temática de teoría de sistemas (Rodin, et al.). 
En la sociología y en las ciencias sociales en general, una concepción primitiva de sistemas, que los restringe a un conjunto determinado de relaciones ordenadas entre las partes de un todo, tiene hasta el día de hoy vigencia. La clásica y reiterada definición de sistema elaborada por Hall y Fagen (1956), en términos de un conjunto de objetos y sus relaciones y las relaciones entre éstos y sus atributos, es la expresión del modelo que antes comentábamos. Esta definición representa un sistema encerrado en sus operaciones internas y casi sin tomar en consideración al ambiente ${ }^{3}$. Similares aplicaciones se encuentran en los trabajos de Talcott Parsons, el cual hizo depender la existencia de un sistema a la satisfacción de los por él denominados prerrequisitos funcionales ${ }^{4}$.

Si bien en estas concepciones sociológicas no hay, estrictamente hablando, puesta en juego una noción cabal de sistemas cerrados, existe, sin embargo, una fuerte concentración en la atención de las relaciones que ocurren al interior de los sistemas. Este nivel de desarrollo de la teoría de sistemas se encuentra fuertemente entroncado con las nociones de totalidad y organicidad. Estos análisis incluyen consideraciones específicas acerca de los elementos o partes constituyentes de los sistemas, pero éstos se dirigen fundamentalmente en dirección de destacar las relaciones de interdependencia que dan lugar al todo. Con ello adquiere relieve, como antes lo mencionáramos, la tarea de definir los límites del tipo de sistemas que nos interesan y el cómo resolver el problema del cambio.

En su utilización concreta, este enfoque, especialmente aplicable al campo de las organizaciones, destaca el viejo principio aristotélico de que la suma de las partes no sería igual al todo, fenómeno conocido actualmente como efecto sinérgico. Sociedades y culturas, así como los grupos, las organizaciones y las interacciones, no serían el producto de la adición simple de sus componentes, sino el resultado de las relaciones que éstos establecen ${ }^{5}$. Sin embargo, la noción de elemento o parte, para la cual está mejor preparado el instrumental de la observación, no pierde en absoluto su vigencia y ocupa un espacio destacado en las descripciones de sistemas. La disposición semiestable de los elementos y sus relaciones pasa a denominarse comúnmente estructura

\footnotetext{
${ }^{3}$ Esta aplicación es de amplio uso en la lingüística. El mismo Saussure estableció frecuentemente analogías entre el lenguaje y el juego de ajedrez, reduciendo ambos a conjuntos de reglas de relaciones posibles entre los elementos y sus atributos que operan dentro de límites determinados (SAUSSURE, 1984, op. cit.).

${ }^{4}$ De acuerdo con la formulación original de T. Parsons, éstos ser ian: la adaptación, el procuramiento de metas, la integración y el mantenimiento de patrones culturales, conocidos también como el esquema AGIL (PARSONS, T., 1961, op. cit.).

${ }^{5}$ Estas afirmaciones son, por cierto, reversibles: la cantidad de estados posibles a ser asumidos por los elementos de un sistema son mucho mayores que los que realmente se actualizan. Los sistemas, una vez constituidos limitan posibilidades, en este sentido son mucho menos que la suma de sus partes. En otras palabras: son siempre menos complejos que sus ambientes.
} 
del sistema, la que, bajo otros términos, sería la condición alcanzada mediante la interdependencia o, en otras palabras, el origen de la estabilidad de los sistemas. Las regularidades y continuidad temporal de la dinámica que los caracteriza se denominan procesos sistémicos: se acepta además que, en base a una tendencia a la especialización, los sistemas pueden irse diferenciando, primero internamente en subsistemas y luego del propio sistema-madre, originando nuevas variedades socioculturales.

Todas estas nociones condujeron rápidamente al problema de la determinación de los límites de un sistema, cuya solución no es siempre convenientemente aclarada. Frente a ello, la teoría en curso explora un nuevo concepto: la recursividad (BeER). Esto es: sistemas viables podrían considerarse parte de otros. A su vez, pueden estar compuestos por subsistemas y así sucesivamente. Los límites de esta descomposición llegarán hasta un nuevo nivel de emergencia. Ello sería la indicación de un cambio cualitativo. Se trata, por ejemplo, del paso de células a organismos hasta la emergencia de sistemas síquicos y posteriormente socioculturales. Este fenómeno está muy bien descrito por Morin (1974), cuando indica que la emergencia de un sistema implica la posesión de cualidades y atributos que no se sustentan en las partes aisladas y que, por otro lado, los elementos o partes de un sistema actualizan cualidades y propiedades que sólo son posibles en el contex to de un sistema dado. En otras palabras, las características que observamos en los sistemas no son reducibles ni a sus elementos ni a los a tributos de éstos, sino que se deben al tipo de relaciones selectivas que se dan en su contexto sistémico. Esto significa que las propiedades inmanentes de los componentes de un sistema tampoco pueden aclarar su emergencia. Se habla en este plano de la existencia de condiciones de autocatálisis de sistemas socioculturales.

Existen también esfuerzos para aclarar el problema de la indeterminación de límites en los sistemas que van en otra dirección, esto es, aplicando a los sistemas la noción de unidades de análisis, es decir, una forma de operacionalismo en la cual los sistemas son constructos; su identidad y límites quedan en manos del investigador y de sus propósitos o, en el mejor de los casos, están sujetos a un complejo aparataje deductivo, como sucede en el caso de la teoría estructural-funcionalista y en el materialismo histórico. Cabe destacar que el enfoque de sistemas es radicalmente distinto a estas teorías, en la medida que no recurre a formulaciones deterministas o causalistas como es lo característico de ambas perspectivas.

Un importante paso en el desarrollo de la teoría de sistemas se alcanza cuando la observación de los sistemas biológicos y el desarrollo de la cibernética (WIENER) ${ }^{6}$ conducen a la superación de la concentración en las relaciones al interior de los sistemas y a una consecuente

\footnotetext{
${ }^{6}$ Cibernética es un concepto que designa la ciencia que se ocupa de los modos de gobierno. Etimológicamente significa "piloto" o "gobernar y dirigir un navio".
} 
aceptación de los presupuestos que apuntaban al manejo de las nociones de Bertalanffy, especialmente en lo referido a la apertura de los sistemas vivos. La relación todo-partes, que entrababa la teoría con la observación, pasa a ser sustituida por la de sistema-ambiente. Diversos componentes del orden de complejidad teórica y conceptual acompañan este cambio.

El equilibrio y la sobrevivencia de los sistemas se ponen en estrecha relación con las condiciones que presenta el ambiente. Los sistemas son visualizados como expuestos a las contingencias de su entorno y éste es visto como una fuente de perturbaciones que deben y pueden ser controladas. El éxito de un sistema pasa a medirse en la perduración de su estructura. Su función básica y primordial es la denominada morfostasis. Las relaciones internas adquieren su sentido, ahora, en relación con el ambiente, son el instrumental requerido para su sobrevivencia. El problema central, para la ciencia teórica y aplicada, pasa a ser la viabilidad de los sistemas, es decir, su capacidad de adaptación. En este sentido, los sistemas a través de su organización contrarrestarían las tendencias entropicas ${ }^{7}$; sus mecanismos serían el establecimiento y control selectivo de sus intercambios con el ambiente.

En esta última dirección, los sistemas son evaluados en términos de su adecuación al ambiente. Pronto se descubre que para estos fines los sistemas pueden desarrollar respuestas que llegarían, incluso, a modif icar o ampliar su propia estructura, aumentando, por ejemplo, su natural variedad interna (AsHBy), incrementando con ello su complejidad. Paralelamente, son observados y analizados el desarrollo y existencia de mecanismos homeostáticos o de autorregulación que acaecen corrientemente en los sistemas biológicos. La sobrevivencia, en estos ámbitos, implica relacionarse con el ambiente en términos de un intercambio. En este punto son desarrollados conceptos tales como: energía, materia e información. Este enfoque se aplicó especialmente en la denominada sociología de las organizaciones (KATZ y KAHN).

La estructura de un sistema empieza a ser tratada como una variable, se generaliza la aplicación del esquema input-output, se desarrolla el concepto de retroalimentación negativa, que alude a los procesos de corrección de las desviaciones a partir de la captación de informaciones ambientales, el feedbak pasa a ser concebido como un mecanismo de control. Este tipo de análisis traspone los límites de la teoría y del análisis científico y empieza a ser aplicado decididamente en la construcción de sistemas artificiales. Así, cierto tipo de organizaciones pasan a ser definidas en cuanto sistemas sociotécnicos de hombres y máquinas, desarrollándose con ello una modalidad de ingeniería social. Este proceso se complica con la asimilación de los nuevos avances de la cibernética (Wiener) de la teoría de la información (Shannon

\footnotetext{
${ }^{7}$ La entropia define un estado de lo probable. Su característica es el desorden, la desorganización. Este fenómeno se explica por la segunda ley de la termodinámica.
} 
y Weaver), de la teoría de los juegos (Von Neumann y Mor.

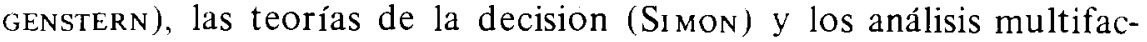
toriales que permiten relacionar grandes cantidades de datos.

La relación entre un sistema y su ambiente empieza a ser manejada a base del concepto de complejidad, problema que nos remite a la cantidad de elementos dentro de un sistema, sus posibles relaciones y, finalmente, a las relaciones entre estas relaciones. En este sentido el ambiente es considerado siempre como más complejo que el sistema y la función básica para éste pasa a ser el control y reducción de esa complejidad, asunto que se realiza por medio de mecanismos selectivos, pues es evidente que ningún sistema puede relacionarse con su ambiente de igual a igual. En esa perspectiva, se hacen notorias las reales posibilidades de los sistemas, las cuales sobrepasan su trivialización en términos de relaciones input-output y del set de mecanismos de control y corrección que antes se presuponían. Este desarrollo incorpora nuevos conceptos a la teoría, los cuales trastruecan nuestra visión de los sistemas, como por ejemplo, la retroalimentación positiva (Mayurana), elaboración de decisiones (Deutsch), y se rescata el concepto de equifinalidad elaborado con mucha anterioridad por Bertalanffy. Junto a ello son reconocidas las posibilidades con que cuentan los sistemas para modificar sus propias estructuras, esto es, la morfogénesis (BUCKLEY). Estas nociones, incorporadas a la idea de sistemas abiertos, son aplicadas a diversos ámbitos de la realidad sociocultural, destacándose, por cierto, en el campo de las organizaciones formales.

El último nivel alcanzado por la teoría de sistemas en el campo de las ciencias sociales ha sido, sin duda, la incorporación a ella de los resultados de las investigaciones realizadas en Chile por el biólogo Humberto Maturana y sus colaboradores en la Facultad de Ciencias de la Universidad de Chile, específicamente, como ya antes lo habíamos mencionado, la noción de autopoiesis, concepto frente al cual reaccionó favorablemente Niklas Luhmann en Alemania ${ }^{8}$. Con este descubrimiento científico tanto la concepción de sistemas cerrados como la de sistemas abiertos son definitivamente sobrepasadas, abriendo paso a las nociones de autorreferencialidad y de autorreproducción de los elementos que componen un sistema.

\footnotetext{
${ }^{8}$ Niklas Luhmann $(61)$ es uno de los más importantes científicos sociales contemporáneos. Sus méritos lo han hecho acreedor de múltiples distinciones, la última de las cuales es el Friedrich Hegel-Preis (1988). Actualmente se encuentra en la Universidad de Bielefeld en la República Federal Alemana. La obra de Luhmann se compone de más de treinta libros y cientos de artículos publicados en las más prestigiosas revistas especializadas del mundo académico. Sus estudios son vastamente citados en la literatura moderna de su especialidad. Lamentablemente se cuenta, hasta el momento, con pocas traducciones de su obra al idioma castellano, entre otras: La Ilustración sociológica y otros ensayos (1973), Fin y racionalidad en los Sistemas (1983a), Sistema Juridico y Dogmática Juridica (1983b) y El amor como pasión (1985).
} 
Mediante estas nuevas concepciones teóricas pueden ser entendidos más claramente algunos vacíos que no fueron convenientemente aclarados con las anteriores nociones. Específicamente, como señala Luhmann, se abre la posibilidad de entender mejor el surgimiento y las relaciones entre procesos sistémicos tales como la autoorganización y muy en especial, para el caso de los sistemas socioculturales y síquicos: la autorreflexión. Este último fenómeno, muy cercano a los enunciados tautológicos, permite, por ejemplo, explicar relaciones tales como amar el amor, creer en la creencia, comunicar comunicaciones, valorar valores, etc.

En términos meramente formales, un sistema autopoiético se puede definir como un sistema, cuyos elementos, de los cuales se compone, a través de las relaciones entre esos elementos, se autorreproducen. El concepto de autopoiesis fue desarrollado y presentado por primera vez, en el mundo científico, por Maturana (1973); su raíz etimológica proviene del griego y se descompone en autos que equivale a si mismo y poein, que corresponde a hacer. Sistemas autopoiéticos son, por tanto, sistemas que se hacen a sí mismos? .

La teoría de la autopoiesis no es meramente una teoría biológica, tomada en préstamo por nuestras disciplinas, sino que es una respuesta frente a dos problemas fundamentales: por un lado permite caracterizar a los sistemas vivos, incluyendo en ellos el mundo de lo síquico y de lo sociocultural, y, por otro, proporciona una perspectiva epistemológica que aborda el problema de la generación del conocimiento. En tanto paradigma científico, esta teoría es pospositivista y posempiricista, válida tanto para las ciencias naturales como para las ciencias sociales. En palabras de Luhmann, se trataría de una verdadera revolución en el campo de la epistemología. Y no solamente este científico alemán ha sido resonante ante esta nueva concepción; figuras de primera línea en el debate académico actual tales como el cibernético británico Gordon Pask (1981), Milan Zeleny (1981), Ranulph Glanville (1981) y Heinz von Foerster (1984), son algunos de sus principales adherentes. El ya citado S. Beer plantea incluso que toda institución social cohesiva tendría que ser un sistema autopoiético; ello incluye, por ejemplo, una empresa, una universidad, un hospital, etc. (1980).

La noción de autopoiesis sustituye a la primitiva noción de autonomía sistémica. Los sistemas socioculturales son, en este sentido, concebidos como cerrados; en lo que respecta a la reproducción de sus elementos, son autorreferenciales, dada su capacidad para autoobservarse, autodescribirse y autorreflexionarse. Esta nueva teoría llena también un vacío con respecto al problema de la historia y el cambio,

\footnotetext{
${ }^{9}$ Para reconocer la aplicación del concepto de autopoiesis a los sistemas sociales resulta conveniente distinguir algunas diferencias entre la perspectiva de Maturana con respecto a la de Luhmann, asunto que ha sido tratado en un reciente trabajo (RodRiGUEZ, D., 1987, op. cit.).
} 
pues ella permite ser relacionada mejor con los procesos evolutivos y explicar los mecanismos para la construcción y la perduración de sistemas. Los conceptos angulares de los modelos teóricos precedentes, tales como ajuste, adaptación o equilibrio, son dejados de lado. De igual manera, la incógnita del porqué un gran número de sistemas permanecen a lo largo del tiempo aparentemente inmutables, a pesar de las grandes variaciones que ocurren en sus entornos, puede ser mejor comprendida. Sus proyecciones, por ejemplo, para los estudios acerca de la difusión o rechazo de innovaciones son insospechadas.

Autopoiesis aplicada a los sistemas socioculturales significa que todo lo que en un sistema funciona recibe su identidad a través del sistema mismo, no solamente en lo que se refiere a su estructura y procesos, sino además en lo que respecta a la propia reproducción de los elementos que los constituyen. De acuerdo a lo planteado por Luhmann, los elementos que componen un sistema societal son las comunicaciones, para las organizaciones se trata de las decisiones y para los sistemas de interacción las acciones tematizadas (LUHMANN, N.). Este nuevo enfoque en la teoría de sistemas implicaría, entre otras cosas, la innovación de considerar la diferenciación entre sistema y entorno en los sistemas socioculturales, como resultado de una clausura autorreferencial con respecto al entorno, lo cual, a su vez, es condición para su apertura. Esta limitación está implicada en la capacidad selectiva de resonancia frente al entorno, la cual es equivalente con la diferenciación misma de los sistemas. En realidad, cuando un sistema no puede filtrar los sucesos de su entorno, no es un sistema propiamente tal.

Naturalmente, los sistemas socioculturales, en cuanto autopoiéticos, y como tales representados como cerrados, autónomos y autorreferenciales, no permiten negar la existencia de un ambiente que les proporciona la energía y la materia para su construcción, tan sólo se trata de que sus elementos y relaciones, esto es, lo que los distingue del ambiente, una vez constituido el sistema, son productos exclusivos de sus operaciones internas. En este último sentido, no hay lugar para la aplicación mecánica del esquema input-output, modelo que sólo es factible de aplicar en términos de metodología de análisis en sistemas cuyas funciones y organización se encuentran, a su vez, determinadas por otros sistemas y cuyo tipo real lo constituyen las máquinas y los complejos sociotécnicos que han surgido de la inventiva humana.

Este nuevo paradigma para el análisis de sistemas no solamente ha afectado la concepción que se tenía acerca de sus objetos, sino, además, las ideas acerca de la misma actividad científica. La teoria se entiende a sí misma como autorreferencial, se piensa a sí misma en cuanto se autoobserva. En ese contexto las distinciones que se hacen con respecto a la diferenciación entre sistema y ambiente son el correlato de la posibilidad de observación de esa distinción. Todo lo anterior conlleva un complicado aparataje conceptual de distinciones, en el cual es difícil encontrar elementos fijos o puntos de referencia 
estables. Los instrumentos clásicos del análisis científico, esto es, la separación entre sujeto y objeto, los esquemas de causalidad y el instrumentario metodológico que los acompañan, quedan, bajo esta perspectiva, abruptamente relativizados, pasando a ser equivalentes alternativos frente a otro tipo de relaciones, como por ejemplo: las circulares, las recursivas, las intermitentes, etcétera.

Esta nueva teoría es presentada por Luhmann como ... un extenso, elegante y económico instrumento de aclaración para los aspectos positivos o negativos de la sociedad modema (1985a); podría añadirse, de ninguna manera sencillo. Como el mismo autor lo indica, esta teoría no solamente debe ser compleja, sino que debe llegar a ser cada vez más compleja, de esta manera corresponderá a la complejidad de su objeto -el individuo, la sociedad y la cultura- y podrá decir algo más que sencillas simplificaciones. En compensación, los interesados en aplicar este tipo de teorías deben imbuirse en un ambiente conceptual muy abstracto y aparentemente árido, pero cuyos rendimientos son insospechados.

En una teoría que se aplica a sí misma, resulta indispensable definir el punto de vista que se utiliza en los análisis. Así, el científico debe ser considerado como un caso especial de observador externo; en concreto, un observador de observadores. Para el caso de la sociología, su tarea es describir la autodescripción de la sociedad. En otras palabras: una observación societal de segundo orden. Lo mismo ocurre para el antropólogo interesado en los fenómenos de la cultura ${ }^{10}$.

Como tanto el antropólogo y el sociólogo se valen en sus estudios de la observación, ésta, a su vez, debe ser concebida como una operación de diferenciación. En efecto, la diferenciación es el único recurso que permite ganar información. El valor y profundidad de la observación dependen abiertamente de la calidad de las distinciones que pueden realizarse sobre un ambiente determinado. El ambiente es para el sistema científico, como para cualquier otro sistema, una premisa dependiente de sus propias operaciones internas: un sistema sólo puede ver lo que puede ver, y no puede ver lo que no puede ver. El papel de la teoría queda claramente perfilado a partir de lo implicado en la aseveración precedente. El antropólogo y el sociólogo, en sus tareas investigativas, configuran sistemas que miran observadores. Frente a ellos tienen la ventaja de poder captar el esquema de observación del cual no son conscientes sus observados. Esta idea estaba, con anterioridad, presente en otras teorías concurrentes, enmarcada en conceptos tales como alienación, funciones latentes o inconsciente. Queda en evidencia, por tanto, que las metas de la investigación científica distan mucho de la mera reproducción de la realidad observada, por más prolija que ésta sea. En todo caso la teoría de sistemas, en su versión social y cultural, posibilita una reconstrucción, en el lenguaje científi-

\footnotetext{
${ }^{10}$ Esta perspectiva guarda estrecha relación con el enfoque émico que prevalece en la Antropología Cultural contemporánea (ARNOLD, M., op. cit.).
} 
co, de estructuras y procesos que acontecen efectivamente; en este plano está fuera de lugar la discusión acerca de su competencia en cuanto instrumento intelectual para abordar la realidad que nos interesa en cuanto cientistas sociales.

\section{Bibliografía}

Arnold, M. "Exposición crítica sobre las perspectivas teóricas de la Antropología Cognitiva". Revista Chilena de Antropologia, Santiago, N $^{\circ}$ 6, 1987, pp. 13-25.

Ashby, R. An Introduction to Cybernetics. New York: Wiley, 1958.

Beer, S. Decisions and Control. London, Wiley and Sons Inc., 1970.

Beer, S. "Preface", en H. Maturana et al., Autopoiesis and cognition: The Realization of the Living, Dordrecht - Boston - London, Reidel.

Bertalanffy, L. von "The Theory of Open Systems in Physics and Biology", en Science, III, pp. 23-29, 1950.

Buckley, W. La sociologia y la teoria moderna de los sistemas. Buenos Aires, Ed. Amorrortu, 1973.

Deutsch, K. The Nerves of Government: Models of Political Communication and Control. New York, 1963.

Foerster, H. von. Observing Systems. Seaside (Cal.), Intersystems Publications, 1984.

Glanville, R. "The Same is Different", en M. Zeleny (ed.) Autopoiesis. A Theory of Living Organization, New York, Oxford, 1981.

Hall, A. y Fagen, R. "Definition of System", en General System 1, pp. 18-28, 1956.

Katz, D. y Kahn, R. The social Psychology of Organizations, New York, Wiley and Sons Inc., 1966.

Kuhn, T. Die Struktur wissenschaftlicher Revolutionen, Frankfurt, Suhrkamp, 1973.

Luhmann, N. La ilustración sociológica y otros ensayos. Buenos Aires, Ed. SUR. 1973.

Luhmann, N. "Interaktion, Organisation, Gesellschaft", en Soziologische Aufklärung, Bd. 2, Opladen, Westdeutscher Verlag, 1975a.

Luhmann, N. Fin y Racionalidad en los Sistemas, Madrid, Ed. Nacional, 1983a.

Luhmann, N. Sistema Juridico y Dogmática Juridica, Madrid, Centro de Estudios Constitucionales, $1983 \mathrm{~b}$.

Luhmann, N. Soziale Systeme. Grundriss einer allgemeinen Theorie, 2. Aufl., Frankfurt/M., Suhrkamp, 1985a.

Luhmann, N. El amor como pasión, Barcelona, Ediciones Península, 1985 b.

Maturana, H. y Varela, F. De máquinas y seres vivos, Santiago, Editorial Universitaria, 1973.

Mayurana, M. "The second Cybernetics: Deyiation Amplifying Mutual Causal Processes". American Scientist, No 1, 1963, pp. 164-179.

Merton, R. Teoria y Estructura Sociales. México, Fondo de Cultura Económica, 1970.

Morin, E. "Complexity", en International Social Science, 26, Paris, 1974, pp. $55-$ 582.

Neumann, J. von y Morgenstern, $\mathbf{O}$. Theory of Games and Economic Behavior, Princeton, Princeton University Press, 1947. 
Parsons, T. "An Outline of the Social System", en Theories of Society, T. Parsons et al., Glencoe, 1961.

Pask, G. "Organizational Closure of Potentially Conscious Systems", en M. Zeleny (ed.), Autopoiesis. A Theory of Living Organization, New York, Oxford, 1981 .

Quiroz, D. Antropologia de los sistemas culturales: una aproximación epistemológica y metodológica, Tesis de Antropología, Universidad de Chile, 1978.

Rodin, M. et al. "System Theory in Anthropology", Current Anthropology, vol. $19, \mathrm{~N}^{\circ} 4$, December 1978, pp. 747-762.

Rodríguez, D. "Teoría de sistemas: situación actual", Estudios Sociales, Santiago, No 43, pp. 15-32, 1985.

Rodríguez, D. "Elementos para una comparación de las teorías de Maturana y Luhmann", Estudios Sociales, Santiago, N 54, pp. 9-30, 1987.

Saussure, F. Curso de Lingüística General, Buenos Aires, 1984 (1916).

Shannon, C. y Weaver, w. The Mathematical Theory of Communication, Urbana, The University Press, 1949.

Simon, H.A. "Theories of Decision-Making in Economics and Behavioral Science", The A merican Economics Review, 49, pp. 253-283, 1959.

Wiener, N. The Human Use of Human Beings: Cybernetics and Society, Garden City, New York, 1954.

Zeleny, M. Autopoiesis. A Theory of Living Organization, New York, Oxford, 1981 . 